C'est le cas du polyéthylène qui peut se présenter sous la variété " haute densité » ou la variété " basse densité ». Les critiques concernant l'étanchéité des feuilles s'adressent au produit "basse densité » (la variété " haute densité » est cinq à six fois plus imperméable).

D. - Il faut considérer l'intensité au contact du produit alimentaire avec la matière plastique.

Il est bon de connaître :

10 La surface de contact par gramme de produit;

2 - La durée du contact;

$3^{\circ}$ Les températures extrêmes.

\title{
Conclusion
}

En conclusion, sur le plan technologique, l'emploi des plastiques dans l'industrie laitière a déjà donné lieu à de nombreuses applications qui se sont révélées intéressantes.

D'autre part, l'exploitation méthodique des possibilités d'emploi des matières plastiques existantes pour le traitement du lait ne peut pas, aujourd'hui, être considérée comme terminée et il est à peu près certain que d'autres verront le jour dans les années qui viennent.

Enfin, il est très vraisemblable que de nouvelles matières plastiques pourront s'ajouter utilement à la liste actuelle.

\section{ASPECTS PARTICULIERS DE LA PASTEURISATION DU MÉLANGE DE CRÈmE GLACÉE (1)}

par

\author{
Joseph IOBIAS et G. A. MUCK
}

On admet généralement que la pasteurisation appliquée aux produits laitiers a comme but premier, la destruction des bactéries pathogènes, par un traitement à la chaleur approprié. Cependant cet exposé est incomplet, car ce n'est pas dans ce seul but de rendre les produits sains et inoffensifs que l'on utilise la pasteurisation. Une meilleure conservation, une protection contre l'oxydation du gras et le contrôle de la stabilité à la chaleur sont autant d'exemples des nombreux services que peut rendre la pasteurisation.

Dans la préparation des mélanges de crème glacée, la pasteurisation facilite la dissolution des ingrédients tels que la poudre

(1) Le Québec Laitier et Alimentaire, juin 1964. 
de lait, le sucre, les stabilisants et les émulsifs, porte le mélange à la température d'homogénéisation, favorise l'hydratation des constituants colloïdaux du mélange, tels que les protéines et les stabilisants. Cette dernière fonction est probablement l'une de celles qui est la moins bien comprise.

\section{TABLEAU 1}

EFEET DU TRAITEMENT A LA CHALEUR

SUR LA VISCOSITÉ BASIQUE DES MÉLANGES NON STABILISÉS

Viscosité après traitement à la chaleur à différentes températures

\begin{tabular}{|c|c|c|c|c|c|c|}
\hline & $200^{\circ} \mathrm{F}$. & $230^{\circ} \mathrm{F}$. & $260^{\circ} \mathrm{F}$. & $275^{\circ} \mathrm{F}$. & $290^{\circ} \mathrm{F}$. & $305^{\circ} \mathrm{F}$. \\
\hline Mélange de crème glacée & 19,8 & 18,7 & 26,4 & 31,6 & 31,5 & 31,1 \\
\hline $\begin{array}{c}\text { Mélange lait glacé (Ice } \\
\text { Milk) ..................... }\end{array}$ & 13,7 & 13,8 & 18,2 & 24,1 & 25,4 & 23,7 \\
\hline
\end{tabular}

Il semble logique de penser, qu'il est désirable de retrouver dans le mélange une grande capacité d'absorption pour l'eau, mais malheureusement, il n'existe pas d'épreuve à la fois simple et positive pour déterminer l'eau liée, et on utilise généralement une épreuve de viscosité à la place. Voici au tableau 1, une illus. tration de l'effet du traitement à la chaleur sur la viscosité basique d'un mélange non stabilisé de crème glacée contenant 12 p. 100 de gras, 11 p. 100 de solides non gras, 15 p. 100 de sucrose et d'un mélange non stabilisé de "lait glacé » contenant 5 p. 100 de gras, 14 p. 100 de solides non gras, 12 p. 100 de sucrose et 6 p. 100 de sirop de maïs. Le chauffage d'une durée de 6 secondes, sans retenue, s'est opéré dans un préchauffeur tubulaire.

L'augmentation de viscosité avec l'augmentation de température est due semble-t-il, à l'interaction des protéines du lait, du sucre et du gras, résultant en un système qui possède de plus grandes particules, dû̀ à une hydratation plus poussée.

Les stabilisants ont aussi un effet identique tel que nous l'indi. quent des données du tableau 2. Le pourcentage de la concentration de la gomme dans la phase aqueuse du mélange de crème glacée fut le même dans chaque cas que dans la solution aqueuse de gomme. En empêchant toute interaction, on devrait ainsi s'attendre à ce que la viscosité des mélanges contenant de la gomme soit en gros, la somme des viscosités du système aqueux de la gomme et du mélange non stabilisé (tableau 1). Les données indiquent clairement qu'une relation si simple n'existe pas et que les interactions avec les solides du mélange ont probablement lieu à la suite du traitement à la chaleur. 


\section{TABLEAU 2}

EFFET DU TRATTEMENT A LA CHALEUR SUR LA VISCOSITÉ DES SYSTÈMES FIXÉS

Viscosité après traitement à la chaleur à différentes températures

\begin{tabular}{|c|c|c|c|c|c|c|}
\hline Produit & $200^{\circ} \mathrm{F}$. & $230^{\circ} \mathrm{F}$. & $260^{\circ} \mathrm{F}$. & $275^{\circ} \mathrm{F}$. & $290^{\circ} \mathrm{F}$ & $305^{\circ} \mathrm{F}$. \\
\hline Carrageenin $(0,04$ p. 100$)$ & 13,7 & 3,7 & 3,8 & 3,7 & 3,8 & 3,8 \\
\hline $\begin{array}{l}\text { CMC }(0,16 \text { p. } 100)(1) \ldots \\
\text { Gomme de Locuste }\end{array}$ & 14,4 & 14,0 & 14,1 & 14,1 & 13,9 & 14,0 \\
\hline$(0,14$ p. 100$) \ldots \ldots \ldots$ & 7,5 & 8,4 & 8,4 & 8,6 & 8,0 & - \\
\hline $\begin{array}{l}\text { Algine }(0,373 \text { p. } 100) \\
\text { Mélange Crème glacée et }\end{array}$ & 3,3 & 3,3 & 3,3 & 3,3 & 3,3 & 3,3 \\
\hline Carrageenin .......... & 22,6 & 26,6 & 28,4 & 64,5 & 47,0 & 46,9 \\
\hline $\begin{array}{l}\text { Mélange C.G. + C.M.C. } \\
\text { Mélange }+ \text { Gomme Lo- }\end{array}$ & 44,6 & 47,9 & 49,6 & 59,3 & 53,9 & 52,8 \\
\hline custe $\ldots \ldots \ldots \ldots \ldots$ & 39,1 & 40,4 & 45,3 & 55,4 & 51,3 & 52,3 \\
\hline Mélange + Algine $\ldots .$. & 67,4 & 64,7 & 71,8 & 67,1 & 79,4 & 80,0 \\
\hline
\end{tabular}

(1) C.M.C. : Carboxy Methy Cellulose.

L'augmentation de la viscosité du mélange due au traitement à la chaleur a été appelée stabilisation à la chaleur ou surchauffage et a induit les gens à conclure que l'on peut ajouter moins de stabilisant quand on pasteurise à de plus hautes températures. Quelques questions demeurent sans réponses, cependant telles que, comment se compare la stabilisation à la chaleur à la stabilisation conventionnelle et jusqu'à quel point peut-on réduire le stabilisant avec une composition de mélange et un traitement à la chaleur donnés.

Des études antérieures sur la pasteurisation continue des mélanges de crème glacée poursuivies à l'Université de l'Illinois ont révélé que la pasteurisation à haute température (H.T.S.T.) donne la même destruction de la phosphatase et des organismes bactériologiques que la pasteurisation conventionnelle à $155^{\circ} \mathrm{F}$ pour 30 minutes mais n'a pas réussi à donner un mélange capable de congeler en une crème glacée d'un corps et d'une texture équivalente. Alors que la température était augmentée, on notait un changement marqué dans le corps et la texture. Un nombre de manufacturiers de crème glacée ont rapporté des expériences similaires quand les normes de pasteurisation furent fixées à $175^{\circ} \mathrm{F}$ pour 25 secondes parce qu'ils furent incapables de reproduire le corps et la texture de la crème glacée qu'ils obtenaient avec leur système à basse température à moins d'augmenter la quantité de stabilisant ou d'élever la température. Ces faits ont porté quelques manufacturiers à expérimenter les températures de chauffage dans la marge de 200 à $300^{\circ} \mathrm{F}$ avec des retenues jusqu'à 
40 secondes, communément appelés pasteurisations à très hautes températures (U.H.T.).

Comme on pouvait s'y attendre le traitement à la chaleur des mélanges de crème glacée a un effet sur la saveur, le corps et la texture de la crème glacée. Le principal défaut de saveur a été appelé soit saveur de cuit, de brûlé, de caramel. Son intensité varie avec la température et la retenue employées mais il dépend aussi de facteurs tels que la composition du mélange, le chauffage des ingrédients, les caractéristiques du transfert de la chaleur de l'outillage et l'absence de particules brûlées sur les surfaces métalliques. Du point de vue du consommateur, ces opinions varient qu'à la signification de saveur de cuit mais tous les efforts pour obtenir un maximum de stabilité à la chaleur résultent généralement en une intensité accrue de la saveur de cuit.

Les expériences ont indiqué que lorsque la stabilisation à la chaleur aide à la production d'une crème glacée ayant un corps et texture désirables, elle ne fournit pas la même protection contre les variations brusques de température comme le font certains stabilisants. Quand vous utilisez la très haute température, les réductions dans l'emploi de stabilisants doivent être faites avec circonspection et seulement lorsqu'on est bien sûr que la qualité du produit n'en sera pas affectée et se maintiendra durant l'entreposage de la crème glacée.

Le tableau 3 nous indique les résultats obtenus lors du jugement du corps et la texture de la crème glacée fabriquée à partir du même mélange soumis à différentes températures. Le préchauffage à très haute température a été fait dans un préchauffeur à plaques mais sans retenue. Le mélange contenait 12 p. 100 de gras, 11 p. 100 de solides non gras et 15 p. 100 de sucrose et fut stabilisé avec un mélange de corrageenin de carboxy-méthyl-cellulose et un émulsif. 30 points ont été adoptés comme pointage pour un corps et texture parfaits.

TABLEAU 3

EFFET DE LA TEMPÉRATURE DE PASTEURISATION SUR LE CORPS ET LA TEXTURE DE IA CR ÈME GLACÉE

\begin{tabular}{|c|c|c|c|c|}
\hline & $\begin{array}{l}60^{\circ} \mathrm{F} \text {. } \\
30 \mathrm{~min} \text {. }\end{array}$ & $220^{\circ} \mathrm{F}$. & $240^{\circ} \mathrm{F}$. & $260^{\circ} \mathrm{F}$. \\
\hline 4 jours $\ldots \ldots \ldots \ldots \ldots \ldots \ldots$ & 30 & 30 & 30 & 30 \\
\hline 4 semaines $\ldots \ldots \ldots \ldots \ldots \ldots \ldots$ & 30 & 30 & 30 & 30 \\
\hline 4 semaines + variations $\ldots \ldots \ldots$ & 29,8 & 29,75 & 29,9 & 29,9 \\
\hline 5 semaines $\ldots \ldots \ldots \ldots \ldots \ldots$ & 30 & 30 & 30 & 30 \\
\hline 5 semaines + variations temp.. & 29,8 & 29,9 & 29,8 & 29,95 \\
\hline
\end{tabular}


Le tableau ne montre pas les changements qui ont été observés dans le corps et la texture de la crème glacée faite de mélange dont le contenu en stabilisant baisse progressivement. Ces mélanges furent stabilisés avec un mélange de CMC et corrageenin et chauffés à $240^{\circ} \mathrm{F}$ sans retenue. La même quantité d'émulsifs fut ajoutée à tous les mélanges.

TABLEAU 4

EFFET DE LA RÉDUCTION DU STABILISANT

SUR LE CORPS ET LA TEXTURE DE LA CRk̀ME GLACÉE

\begin{tabular}{|c|c|c|c|}
\hline & $\begin{array}{c}\text { (Stabilisant) } \\
100 \text { p. } 100\end{array}$ & $\begin{array}{c}\text { (Stabilisant) } \\
85 \text { p. } 100\end{array}$ & $\begin{array}{c}\text { (Stabilisant) } \\
70 \text { p. } 100\end{array}$ \\
\hline $\begin{array}{l}\text { Après } 24 \text { heures } \ldots . . . . \\
\text { Après } 1 \text { semaine } \ldots \ldots \ldots \\
\text { Après } 1 \text { semaine }+ \text { varia- } \\
\text { tions de température .. } \\
\text { Après } 3 \text { semaines } \ldots \ldots \ldots \\
\text { Après } 3 \text { semaines }+\ldots \text { varia- } \\
\text { tions de température .. }\end{array}$ & $\begin{array}{l}29,9 \\
29,9 \\
29,7 \\
29,9 \\
29,5\end{array}$ & $\begin{array}{l}29,9 \\
29,8 \\
29,6 \\
29,9 \\
29,3\end{array}$ & $\begin{array}{l}29,9 \\
29,8 \\
29,6 \\
29,9 \\
29,3\end{array}$ \\
\hline
\end{tabular}

Ces résultats représentent la meilleure stabilité du corps et de la texture observée sur un nombre d'essai. Dans tous les cas les pointages pour le corps et la texture se sont abaissés avec la diminution dans le niveau de stabilisant et dans la plupart des cas plus rapidement que dans les cas décrits dans le tableau 4. La saveur de tous les échantillons est demeurée satisfaisante.

La pasteurisation continue a des avantages définis sur la pasteurisation discontinue particulièrement au point de vue de la production et de l'efficacité de l'utilisation de l'espace. Les coûts de pasteurisation peuvent être réduits en appliquant le principe de la régénération et en obtenant un plus grand rendement par homme/heure.

Ces avantages ne doivent pas être dédaignés mais la relation entre la température de pasteurisation et le pointage pour le corps et la texture devrait être bien compris avant de faire un changement.

\section{L'ALIMENTATION DES BREBIS LAITIÈRES (1)}

\section{par \\ F. BONIOL}

Après trois années d'augmentation, la production totale de lait de brebis au cours de l'année 1963 a baissé d'environ 10 p. 100 par rapport à 1962 .

(1) La Revue de l'élevage, 1964, n० 10, 1039. 